ANNALES

POLONICI MATHEMATICI

$95.1(2009)$

\title{
On solutions of a fourth-order Lidstone boundary value problem at resonance
}

\author{
by MARIUSz Jurkiewicz (Łódź)
}

Abstract. We consider a Lidstone boundary value problem in $\mathbb{R}^{k}$ at resonance. We prove the existence of a solution under the assumption that the nonlinear part is a Carathéodory map and conditions similar to those of Landesman-Lazer are satisfied.

1. Introduction. In this article we deal with the existence of solutions for fourth-order boundary value problems (BVP). We consider the system

$$
\begin{aligned}
&\left(x^{1}\right)^{(4)}-\lambda^{1}\left(x^{1}\right)^{\prime \prime}-\mu^{1} x^{1}=f^{1}\left(t, x^{1}, \ldots x^{k},\left(x^{1}\right)^{\prime \prime}, \ldots,\left(x^{k}\right)^{\prime \prime}\right), \\
& \vdots \\
&\left(x^{k}\right)^{(4)}-\lambda^{k}\left(x^{k}\right)^{\prime \prime}-\mu^{k} x^{k}=f^{k}\left(t, x^{1}, \ldots x^{k},\left(x^{1}\right)^{\prime \prime}, \ldots,\left(x^{k}\right)^{\prime \prime}\right), \\
& x^{1}(0)=x^{1}(1)=\left(x^{1}\right)^{\prime \prime}(0)=\left(x^{1}\right)^{\prime \prime}(1)=0, \\
& \vdots \\
& x^{k}(0)=x^{k}(1)=\left(x^{k}\right)^{\prime \prime}(0)=\left(x^{k}\right)^{\prime \prime}(1)=0,
\end{aligned}
$$

where $f:[0,1] \times \mathbb{R}^{k} \times \mathbb{R}^{k} \rightarrow \mathbb{R}^{k}$ is a Carathéodory map.

In recent years much attention has been given to the above problem for $k=1$ and $\lambda=\mu=0$ by many authors (see $[2,3]$ and references therein). In [4], Youngxiang Li has proved some results for the problem

$$
\begin{aligned}
& x^{(4)}-\lambda x^{\prime \prime}-\mu x=g(t, x), \\
& x(0)=x(1)=x^{\prime \prime}(0)=x^{\prime \prime}(1)=0,
\end{aligned}
$$

where $g:[0,1] \times \mathbb{R} \rightarrow \mathbb{R}$ is continuous and $\lambda>-2 \pi^{2}, \mu \geq-\lambda^{2} / 4$, $\mu / \pi^{4}-\lambda / \pi^{2}<1$. The differential operator which corresponds to the left-hand side of the above equation is always invertible. Here we shall consider the case of a noninvertible operator. In [9] Jolanta Przybycin has obtained the

2000 Mathematics Subject Classification: Primary 34B15; Secondary 35P30.

Key words and phrases: resonance, Lidstone boundary value problem, eigenvalue, Landesman-Lazer condition. 
existence of solutions to a problem similar to the one considered in [4] where $g: \mathbb{R} \rightarrow \mathbb{R}$ does not depend on $t$ and $\lambda \geq 0, \mu \neq-\pi^{2} n^{2}, n=1,2, \ldots$ It is easy to see that for some $\mu, \lambda$ the corresponding differential operator is non-invertible. Her result is a particular case of ours.

Existence of a solution to (1) depends on the vectors $\lambda=\left[\lambda^{1}, \ldots, \lambda^{k}\right]$ and $\mu=\left[\mu^{1}, \ldots, \mu^{k}\right]$; the corresponding differential operator may be invertible or not. Our principal purpose is to examine the latter, so-called resonance case. The first important paper on BVP at resonance appeared in the 70s [7], and this problem has been under examination since then (see [8]).

We start with a crucial definition.

Definition 1.1. A pair $(\lambda, \mu) \in \mathbb{R}^{2}$ will be called a two-dimensional eigenvalue if the homogeneous problem

$$
\begin{aligned}
& x^{(4)}-\lambda x^{\prime \prime}-\mu x=0, \\
& x(0)=x(1)=x^{\prime \prime}(0)=x^{\prime \prime}(1)=0,
\end{aligned}
$$

has a nontrivial solution. The set of all those pairs will be denoted by $\sigma^{2}$.

One can prove that $\sigma^{2}$ is the union of the straight lines $l_{n}$ given by

$$
l_{n}: \mu=n^{2} \pi^{2} \lambda+n^{4} \pi^{4}, \quad n \in \mathbb{N},
$$

that is,

$$
\sigma^{2}=\bigcup_{n \in \mathbb{N}}\left\{(\lambda, \mu) \mid \mu=n^{2} \pi^{2} \lambda+n^{4} \pi^{4}\right\} .
$$

The line $l_{n}$ is tangent to the parabola $\mu=-(\lambda / 2)^{2}$ at $\left(-2 n^{2} \pi^{2},-n^{4} \pi^{4}\right)$. Moreover, $l_{n_{1}}$ and $l_{n_{2}}, n_{1} \neq n_{2}$, intersect at $\left(-\left(n_{1}^{2}+n_{2}^{2}\right) \pi^{2},-n_{1}^{2} n_{2}^{2} \pi^{4}\right)$. Define

$$
\sigma_{1}^{2}:=\sigma^{2} \backslash \bigcup_{\substack{n_{1}, n_{2} \in \mathbb{N} \\ n_{1} \neq n_{2}}}\left\{\left(-\left(n_{1}^{2}+n_{2}^{2}\right) \pi^{2},-n_{1}^{2} n_{2}^{2} \pi^{4}\right)\right\} .
$$

REMARK 1.1. It is easy to check that for each point from $\sigma_{1}^{2}$ (resp. $\left.\sigma^{2} \backslash \sigma_{1}^{2}\right)$ the corresponding eigenspace of the problem (2) is one-dimensional (resp. two-dimensional).

In the next section we consider the simpler case when $(\lambda, \mu) \notin \sigma^{2}$ and $f$ is sublinear. We find a Green map for our problem, and we prove the existence of a solution by using the Schauder fixed point theorem. The case when $(\lambda, \mu) \in \sigma^{2}$ is much more complicated. We examine it for $(\lambda, \mu) \in \sigma_{1}^{2}$ in Section 3. If some conditions of Landesman-Lazer type are satisfied and $f$ is bounded then the problem has a solution.

2. Preliminaries. Assume that $(\lambda, \mu) \notin \sigma^{2}$. Consider the homogeneous equation

$$
x^{(4)}-\lambda x^{\prime \prime}-\mu x=0 .
$$


The set of solutions to (4) is a four-dimensional vector space, denoted by $\mathcal{D}(\lambda, \mu)$. The sets of solutions to the initial value problems

$$
\left\{\begin{array} { l } 
{ x ^ { ( 4 ) } - \lambda x ^ { \prime \prime } - \mu x = 0 , } \\
{ x ( 0 ) = x ^ { \prime \prime } ( 0 ) = 0 , }
\end{array} \text { and } \left\{\begin{array}{l}
x^{(4)}-\lambda x^{\prime \prime}-\mu x=0, \\
x(1)=x^{\prime \prime}(1)=0,
\end{array}\right.\right.
$$

are subspaces of $\mathcal{D}(\lambda, \mu)$, denoted by $\mathcal{D}_{0}(\lambda, \mu)$ and $\mathcal{D}_{1}(\lambda, \mu)$ respectively.

Lemma 2.1. The linear spaces $\mathcal{D}_{0}(\lambda, \mu)$ and $\mathcal{D}_{1}(\lambda, \mu)$ are two-dimensional. Furthermore, $\mathcal{D}(\lambda, \mu)=\mathcal{D}_{0}(\lambda, \mu) \oplus \mathcal{D}_{1}(\lambda, \mu)$.

Let $\widehat{f}:[0,1] \rightarrow \mathbb{R}$ be a bounded continuous function and $(\lambda, \mu) \notin \sigma^{2}$. Consider the problem

$$
\begin{aligned}
& x^{(4)}(t)-\lambda x^{\prime \prime}(t)-\mu x(t)=\widehat{f}(t), \\
& x(0)=x(1)=x^{\prime \prime}(0)=x^{\prime \prime}(1)=0 .
\end{aligned}
$$

By Lemma 2.1, there exist linearly independent solutions $\alpha_{1}, \alpha_{2}, \alpha_{3}, \alpha_{4}$ to the homogeneous equation corresponding to (5) such that $\alpha_{1}(0)=\alpha_{1}^{\prime \prime}(0)$ $=0, \alpha_{2}(1)=\alpha_{2}^{\prime \prime}(1)=0, \alpha_{3}(0)=\alpha_{3}^{\prime \prime}(0)=0, \alpha_{4}(1)=\alpha_{4}^{\prime \prime}(1)=0$. Thus, the fundamental matrix has the form

$$
A(t):=\left[\begin{array}{cccc}
\alpha_{1}(t) & \alpha_{2}(t) & \alpha_{3}(t) & \alpha_{4}(t) \\
\alpha_{1}^{\prime}(t) & \alpha_{2}^{\prime}(t) & \alpha_{3}^{\prime}(t) & \alpha_{4}^{\prime}(t) \\
\alpha_{1}^{\prime \prime}(t) & \alpha_{2}^{\prime \prime}(t) & \alpha_{3}^{\prime \prime}(t) & \alpha_{4}^{\prime \prime}(t) \\
\alpha_{1}^{(3)}(t) & \alpha_{2}^{(3)}(t) & \alpha_{3}^{(3)}(t) & \alpha_{4}^{(3)}(t)
\end{array}\right] .
$$

The matrix $A$ with the $i$ th column and fourth row deleted will be denoted by $A_{i}$. We check at once that if there exists a solution $x$ to (5)-(6) then

$$
\begin{aligned}
x^{\prime \prime}(t)= & \alpha_{1}^{\prime \prime}(t) \int_{t}^{1} \frac{1}{W(s)} \operatorname{det} A_{1}(s) \widehat{f}(s) d s+\alpha_{2}^{\prime \prime}(t) \int_{0}^{t} \frac{1}{W(s)} \operatorname{det} A_{2}(s) \widehat{f}(s) d s \\
& +\alpha_{3}^{\prime \prime}(t) \int_{t}^{1} \frac{1}{W(s)} \operatorname{det} A_{3}(s) \widehat{f}(s) d s+\alpha_{4}^{\prime \prime}(t) \int_{0}^{t} \frac{1}{W(s)} \operatorname{det} A_{4}(s) \widehat{f}(s) d s .
\end{aligned}
$$

Define $\mathcal{H}:[0,1] \times[0,1] \rightarrow \mathbb{R}$ by the formula

$$
\mathcal{H}(t, s):= \begin{cases}\frac{1}{W(s)}\left(\alpha_{1}^{\prime \prime}(t) \operatorname{det} A_{1}(s)+\alpha_{3}^{\prime \prime}(t) \operatorname{det} A_{3}(s)\right), & 0 \leq t \leq s \leq 1, \\ \frac{1}{W(s)}\left(\alpha_{2}^{\prime \prime}(t) \operatorname{det} A_{2}(s)+\alpha_{4}^{\prime \prime}(t) \operatorname{det} A_{4}(s)\right), & 0 \leq s \leq t \leq 1 .\end{cases}
$$

It is easily seen that $\mathcal{H}$ is continuous. Moreover, we can rewrite (7) as

$$
x^{\prime \prime}(t)=\int_{0}^{1} \mathcal{H}(t, s) \widehat{f}(s) d s .
$$


We equip $\mathbb{R}^{k}$ with the maximum norm $\|\alpha\|=\max \left(\left|\alpha^{1}\right|, \ldots,\left|\alpha^{k}\right|\right)$. Furthermore, $\|\cdot\|_{1}$ is the supremum norm in $C([0,1], \mathbb{R})$, and $\|x\|_{k}=$ $\max \left(\left\|x_{1}\right\|_{1}, \ldots,\left\|x_{k}\right\|_{1}\right)$ is the norm in $C\left([0,1], \mathbb{R}^{k}\right)$.

Suppose that $f:[0,1] \times \mathbb{R}^{k} \times \mathbb{R}^{k} \rightarrow \mathbb{R}^{k}$ is a Carathéodory map, i.e.

(i) the map $[0,1] \ni t \mapsto f(t, x, y)$ is measurable for every $x, y \in \mathbb{R}^{k}$;

(ii) the map $\mathbb{R}^{k} \times \mathbb{R}^{k} \ni(x, y) \mapsto f(t, x, y)$ is equicontinuous for almost all $t \in[0,1]$.

If we define the operation $\bullet: \mathbb{R}^{k} \times \mathbb{R}^{k} \rightarrow \mathbb{R}^{k}$ by $\left[\alpha^{1}, \ldots, \alpha^{k}\right] \bullet\left[\beta^{1}, \ldots, \beta^{k}\right]:=$ $\left[\alpha^{1} \beta^{1}, \ldots, \alpha^{k} \beta^{k}\right]$ then the problem (1) can be rewritten as

$$
\begin{aligned}
& x^{(4)}-\lambda \bullet x^{\prime \prime}-\mu \bullet x=f\left(t, x, x^{\prime \prime}\right), \\
& x(0)=x(1)=x^{\prime \prime}(0)=x^{\prime \prime}(1)=0,
\end{aligned}
$$

where $x=\left[x^{1}, \ldots, x^{k}\right]$ etc. It is easily seen that $\mathbb{R}^{k}$ with the $\bullet$ operation is a Banach algebra.

We shall show that (1) has a solution if the linear differential operator which corresponds to the right hand side of our problem is invertible.

Theorem 2.1. Let $\lambda, \mu \in \mathbb{R}^{k}$ with $\left(\lambda^{j}, \mu^{j}\right) \notin \sigma^{2}$ for $j=1, \ldots, k$. Furthermore, assume that for each $M>0$, there exists $C_{M} \in L^{\infty}\left([0,1], \mathbb{R}_{+}\right)$ such that $\|x\|+\|y\| \leq M$ implies

$$
\|f(t, x, y)\| \leq C_{M}(t)
$$

for almost all $t \in[0,1]$, and

$$
\lim _{M \rightarrow \infty} \frac{\left\|C_{M}\right\|_{L^{\infty}}}{M}=0
$$

Then the problem (1) has a solution.

Proof. From (8) it follows that if $x$ is a solution of (1) then it satisfies the equation

$$
x^{\prime \prime}(t)=\int_{0}^{1} \mathcal{H}(t, s) \bullet f\left(s, x(s), x^{\prime \prime}(s)\right) d s,
$$

where $\mathcal{H}=\left[\mathcal{H}^{1}, \ldots, \mathcal{H}^{k}\right]$ and $\mathcal{H}^{j}$ corresponds to the pair $\left(\lambda^{j}, \mu^{j}\right)$ for $j=$ $1, \ldots, k$. It is obvious that the converse is true as well. Putting $y=x^{\prime \prime}$ one can observe that (1) is equivalent to the system

$$
\begin{aligned}
x(t) & =\int_{0}^{1} G(t, s) y(s) d s, \\
y(t) & =\int_{0}^{1} \mathcal{H}(t, s) \bullet f\left(s, \int_{0}^{1} G(s, p) y(p) d p, y(s)\right) d s,
\end{aligned}
$$


where $G:[0,1] \times[0,1] \rightarrow \mathbb{R}$ has the form

$$
G(t, p)= \begin{cases}p(t-1) & \text { for } 0 \leq p \leq t \leq 1 \\ t(p-1) & \text { for } 0 \leq t \leq p \leq 1\end{cases}
$$

This implies that it is sufficient to prove that the operator $T: C\left([0,1], \mathbb{R}^{k}\right) \rightarrow$ $C\left([0,1], \mathbb{R}^{k}\right)$ defined by the formula

$$
(T y)(t):=\int_{0}^{1} \mathcal{H}(t, s) \bullet f\left(s, \int_{0}^{1} G(s, p) y(p) d p, y(s)\right) d s
$$

has a fixed point.

It is clear that the above definition of $T$ is correct. We may assume that the functions $\left\{C_{M}\right\}_{M>0}$ satisfying (9) have the property, that

$$
\left\|C_{M_{1}}\right\|_{L^{\infty}} \leq\left\|C_{M_{2}}\right\|_{L^{\infty}} \quad \text { for } M_{1}<M_{2} .
$$

By (10) and (11) there exists $R_{1}>0$ such that

$$
\left\|C_{M}\right\|_{L^{\infty}} \leq\left\|C_{R_{1}}\right\|_{L^{\infty}}<\frac{1}{(1+N) N} R_{1} \quad \text { for every } M \leq R_{1},
$$

where $N$ is a common bound of $G$ and $\mathcal{H}$. Therefore, if $\|x\|+\|y\| \leq R_{1}$ then

$$
\|f(t, x, y)\| \leq C_{R_{1}}(t)<\frac{1}{(1+N) N} R_{1}
$$

for almost all $t \in[0,1]$. Set $R:=R_{1} /(1+N)$. Taking any $y \in C\left([0,1], \mathbb{R}^{k}\right)$ such that $\|y\|_{k} \leq R$, we obtain

$$
\|y\|_{k}+\left\|\int_{0}^{1} G(\cdot, p) y(p) d p\right\|_{k} \leq \frac{R_{1}}{1+N}+\frac{R_{1} N}{1+N}=R_{1} .
$$

Therefore, by (12),

$$
\left\|f\left(s, \int_{0}^{1} G(s, p) y(p) d p, y(s)\right)\right\|<\frac{1}{(1+N) N} R_{1} .
$$

Finally, we obtain $\|T y\|_{k} \leq R$.

To sum up, we have shown the existence of $R>0$ such that $T$ maps the ball $\bar{B}_{C}(0, R)$ into itself. From now on, we consider $T$ on this ball only.

We shall show that $T$ is a continuous operator. Choose a sequence $\left\{y_{n}\right\}_{n \in \mathbb{N}} \subset \bar{B}_{C}(0, R)$ such that $y_{n} \rightarrow y_{0}$. Then the sequence $\left\{G(t, s) y_{n}(s)\right\}_{n \in \mathbb{N}}$ is bounded by $R N$ and uniformly convergent to $G(t, s) y_{0}(s)$. The Lebesgue dominated convergence theorem shows that $\left\{\int_{0}^{1} G(t, s) y_{n}(s) d s\right\}_{n \in \mathbb{N}}$ is con- 
vergent. As $f$ is continuous in the second and third variables, we have

$$
\begin{aligned}
\lim _{n \rightarrow \infty} \| f\left(s, \int_{0}^{1} G(s, p) y_{n}(p) d p, y_{n}(s)\right) & \\
& -f\left(s, \int_{0}^{1} G(s, p) y_{0}(p) d p, y_{0}(s)\right) \|=0
\end{aligned}
$$

for almost all $s \in[0,1]$. It is evident that $f\left(s, \int_{0}^{1} G(s, p) y_{n}(p) d p, y_{n}(s)\right), n \in \mathbb{N}$, and $f\left(s, \int_{0}^{1} G(s, p) y_{0}(p) d p, y_{0}(s)\right)$ are bounded by $C_{R+N R}(s)$. Thus

$$
\begin{aligned}
\left\|f\left(s, \int_{0}^{1} G(s, p) y_{n}(p) d p, y_{n}(s)\right)-f\left(s, \int_{0}^{1} G(s, p) y_{0}(p) d p, y_{0}(s)\right)\right\| \\
<2 C_{R+N R}(s),
\end{aligned}
$$

for almost all $s \in[0,1]$. This estimate, (13) and the boundedness of $H$ yield $\left\|T y_{n}-T y_{0}\right\|_{k} \rightarrow 0$ as $n \rightarrow \infty$. This proves the continuity of $T$.

Pick $t_{0} \in[0,1]$ and $\varepsilon>0$. By (9) there exists $\mu>0$ such that

$$
\|f(t, x, y)\| \leq \mu \quad \text { for almost all } t \in[0,1] .
$$

By the continuity of $\mathcal{H}$ there exists $\delta>0$ such that

$$
\left\|\mathcal{H}(t, s)-\mathcal{H}\left(t_{0}, s\right)\right\|<\varepsilon / \mu
$$

for $\left|t-t_{0}\right|<\delta$ and all $s$. Therefore,

$$
\left\|(T y)(t)-(T y)\left(t_{0}\right)\right\| \leq \mu \int\left\|\mathcal{H}(t, s)-\mathcal{H}\left(t_{0}, s\right)\right\| d s \leq \varepsilon .
$$

It is evident that the family $\{T y\}_{y \in \bar{B}(0, R)}$ is uniformly bounded by $R$.

Concluding, the continuous operator $T$ satisfies the assumptions of the Arzelà-Ascoli theorem. Therefore, it is compact, and hence, by the Schauder theorem, it has a fixed point. This completes the proof.

3. Main results. In the previous section we have proved the existence of solutions to (1) in a simpler case without resonance. Now, we deal with the resonance case, which is fundamentally different. We show two results, Theorem 3.1 and Theorem 3.2. The first will be proved only in the case of a scalar equation. The second is a generalization of the second part of Theorem 3.1 and gives the existence of solutions to (1) under some additional assumptions on $\left(\lambda^{i}, \mu^{i}\right), i=1, \ldots, k$. In fact, we have also obtained a vector version of the first part of Theorem 3.1 in $\mathbb{R}^{k}$, but we refrain from presenting it, as it is complicated and we have not found any nonscalar application of it.

We start with some notation. Let

$$
[0,1] \ni t \mapsto \sin n_{0} \pi t
$$


be a basis vector of the eigenspace which corresponds to $(\lambda, \mu) \in \sigma_{1}^{2}$ (recall that $\left.\mu=n_{0}^{2} \pi^{2} \lambda+n_{0}^{4} \pi^{4}\right)$. Let

$$
\Gamma_{n_{0}}^{+}:=\left\{t \in[0,1] \mid \sin n_{0} \pi t>0\right\}, \quad \Gamma_{n_{0}}^{-}:=\left\{t \in[0,1] \mid \sin n_{0} \pi t<0\right\} .
$$

REMARK 3.1. To simplify the notation, we will write $\Gamma^{+}$and $\Gamma^{-}$instead of $\Gamma_{n_{0}}^{+}$and $\Gamma_{n_{0}}^{-}$, respectively. However, it should be remembered that they both depend on $n_{0}$.

Theorem 3.1. Let $(\lambda, \mu) \in \sigma_{1}^{2}$, and let $f:[0,1] \times \mathbb{R} \times \mathbb{R} \rightarrow \mathbb{R}$ be a bounded Carathéodory function with

$$
\lim _{\substack{x \rightarrow+\infty \\ y \rightarrow-\infty}} f(t, x, y)=f_{+}(t), \quad \lim _{\substack{x \rightarrow-\infty \\ y \rightarrow+\infty}} f(t, x, y)=f_{-}(t)
$$

for almost all $t \in[0,1]$.

(i) If the numbers

$$
\begin{aligned}
& \int_{\Gamma^{+}} f_{+}(t) \sin n_{0} \pi t d t+\int_{\Gamma^{-}} f_{-}(t) \sin n_{0} \pi t d t, \\
& \int_{\Gamma^{+}} f_{-}(t) \sin n_{0} \pi t d t+\int_{\Gamma^{-}} f_{+}(t) \sin n_{0} \pi t d t,
\end{aligned}
$$

have opposite signs, then the problem (1) with $k=1$ has a solution.

(ii) If $f_{+}=f_{-}=: f_{0}$ almost everywhere and

$$
\int_{0}^{1} f_{0}(t) \sin n_{0} \pi t d t \neq 0,
$$

then the problem (1) with $k=1$ has a solution.

Proof. Set

$$
\varepsilon_{n}:= \begin{cases}1 / n & \text { if } n \text { is even } \\ -1 / n & \text { if } n \text { is odd }\end{cases}
$$

Consider the sequence of boundary value problems

$$
\begin{aligned}
& x^{(4)}-\left(\lambda+\varepsilon_{n}\right) x^{\prime \prime}-\left(\mu+\varepsilon_{n}\right) x=f\left(t, x(t), x^{\prime \prime}(t)\right), \\
& x(0)=x(1)=x^{\prime \prime}(0)=x^{\prime \prime}(1)=0 .
\end{aligned}
$$

Theorem 2.1 implies existence of a solution $u_{n}$ to the above problem for each $n \in \mathbb{N}$. We shall show that the sequence $\left\{u_{n}\right\}_{n \in \mathbb{N}}$ converges to a solution of (1). The proof will be divided into two steps. First, assume that $\left\{u_{n}^{\prime \prime}\right\}_{n \in \mathbb{N}}$ is bounded, i.e. there exists $M_{2}>0$ such that

$$
\sup _{n \in \mathbb{N}}\left\|u_{n}^{\prime \prime}\right\|_{1}<M_{2} \text {. }
$$


Notice that the sequences $\left\{u_{n}\right\}_{n \in \mathbb{N}},\left\{u_{n}^{\prime}\right\}_{n \in \mathbb{N}},\left\{u_{n}^{(3)}\right\}_{n \in \mathbb{N}},\left\{u_{n}^{(4)}\right\}_{n \in \mathbb{N}}$ are also bounded. Indeed, for $t \in[0,1 / 2]$ the Taylor formula yields

$$
u_{n}(1)-u_{n}(t)=u_{n}^{\prime}(t)(1-t)+u_{n}^{\prime \prime}\left(\theta_{t, n}\right)(1-t)^{2} / 2, \quad \text { where } \theta_{t, n} \in(t, 1),
$$

As $u_{n}(1)=0$, for $n=1,2 \ldots$ we have

$$
\left|u_{n}^{\prime}(t)\right| \leq \frac{\left|u_{n}(t)\right|}{1-t}+\frac{\left|u_{n}^{\prime \prime}\left(\theta_{t, n}\right)\right|(1-t)}{2} .
$$

Since $\left|u_{n}^{\prime \prime}\left(\theta_{t, n}\right)\right|(1-t) \leq M_{2}$ and $1 /(1-t) \leq 2$,

$$
\left|u_{n}^{\prime}(t)\right| \leq\left|u_{n}(t)\right| \cdot 2+\frac{1}{2} M_{2} \text {. }
$$

The above inequality and the fact that $u_{n}(t)=\int_{0}^{t} u_{n}^{\prime}(s) d s$ show that

$$
\left|u_{n}(t)\right| \leq \int_{0}^{t}\left|u^{\prime}(s)\right| d s \leq \frac{1}{4} M_{2}+2 \int_{0}^{t}\left|u_{n}(s)\right| d s .
$$

This and the Gronwall inequality imply that

$$
\left|u_{n}(t)\right| \leq \frac{1}{4} M_{2} \exp \left(2 \int_{0}^{t} d s\right) \leq \frac{1}{4} M_{2} e \quad \text { for } t \in[0,1 / 2]
$$

Similar arguments for $t \in[1 / 2,1]$ give

$$
\left|u_{n}(t)\right| \leq M_{0}:=\frac{1}{4} M_{2} e .
$$

Now, inequality (14) and

$$
\left|u_{n}^{\prime}(t)\right| \leq \frac{\left|u_{n}(t)\right|}{t}+\frac{\left|u_{n}^{\prime \prime}\left(\theta_{t, n}\right)\right| t}{2}
$$

for $t \in[1 / 2,1]$ imply the boundedness of $\left\{u_{n}^{\prime}\right\}_{n \in \mathbb{N}}$ by some $M_{1}$.

The estimate for $\left\{u_{n}^{(4)}\right\}_{n \in \mathbb{N}}$ is a consequence of the boundedness of $f$ on compact sets.

To prove the boundedness of $\left\{u_{n}^{(3)}\right\}_{n \in \mathbb{N}}$ we apply the analogous procedure to the one used for $\left\{u_{n}^{\prime}\right\}_{n \in \mathbb{N}}$. Let $M_{3}$ be the resulting bound of $\left\{u_{n}^{(3)}\right\}_{n \in \mathbb{N}}$.

Thus

$$
M:=\max \left(M_{0}, M_{1}, M_{2}, M_{3}, M_{4}\right)
$$

is a common bound for all the sequences considered.

Fix $r \in\{0,1,2,3\}$ and $t_{0} \in[0,1]$. For any $\varepsilon>0$ let $\delta:=\varepsilon / M$. Then the mean value theorem implies that

$$
\left|u_{n}^{(r)}(t)-u_{n}^{(r)}\left(t_{0}\right)\right|=\left|u_{n}^{(r+1)}\left(\xi_{n, t}\right)\right|\left|t-t_{0}\right| \leq M\left|t-t_{0}\right|<\varepsilon
$$

for $n=1,2, \ldots$ and $\left|t-t_{0}\right|<\delta$. Therefore, the sequences $\left\{u_{n}\right\}_{n \in \mathbb{N}},\left\{u_{n}^{\prime}\right\}_{n \in \mathbb{N}}$, $\left\{u_{n}^{\prime \prime}\right\}_{n \in \mathbb{N}},\left\{u_{n}^{(3)}\right\}_{n \in \mathbb{N}}$ are equicontinuous. From the Arzelà-Ascoli theorem and 
the convergence theorem for derivatives, it now follows that $u_{n}^{(r)} \rightrightarrows u^{(r)}$ for $r=0,1,2,3$ (considering a subsequence if necessary).

From the equality

$$
u_{n}^{(4)}(t)=\left(\lambda+\varepsilon_{n}\right) u_{n}^{\prime \prime}(t)+\left(\mu+\varepsilon_{n}\right) u_{n}(t)+f\left(t, u_{n}(t), u_{n}^{\prime \prime}(t)\right),
$$

the above condition and the continuity of the function $f(t, \cdot, \cdot)$ for almost all $t \in[0,1]$ we see that the sequence $\left\{u_{n}^{(4)}\right\}_{n \in \mathbb{N}}$ converges uniformly to the function $\lambda u^{\prime \prime}(t)+\mu u(t)+f\left(t, u, u^{\prime \prime}\right)$ for almost all $t \in[0,1]$. This together with $u_{n}^{(3)} \rightrightarrows u^{(3)}$ and the convergence theorem for derivatives shows that $u_{n}^{(4)} \rightrightarrows u^{(4)}$ almost everywhere.

Thus, we have shown that

$$
u_{n}^{(r)} \rightrightarrows u^{(r)} \quad \text { for } r=0,1,2,3,4
$$

almost everywhere. This condition enables us to let $n \rightarrow \infty$ to obtain

$$
u^{(4)}-\lambda u^{\prime \prime}-\mu u=f\left(t, u, u^{\prime \prime}\right) .
$$

The above equality means that $u$ is the desired solution.

Now, suppose that $\left\{u_{n}^{\prime \prime}\right\}_{n \in \mathbb{N}}$ is not bounded. Dividing the relevant sequence of equations by $\left\|u_{n}^{\prime \prime}\right\|_{1}$, we get

$$
\frac{u_{n}^{(4)}}{\left\|u_{n}^{\prime \prime}\right\|_{1}}-\left(\lambda+\varepsilon_{n}\right) \frac{u_{n}^{\prime \prime}}{\left\|u_{n}^{\prime \prime}\right\|_{1}}-\left(\mu+\varepsilon_{n}\right) \frac{u_{n}}{\left\|u_{n}^{\prime \prime}\right\|_{1}}=\frac{1}{\left\|u_{n}^{\prime \prime}\right\|_{1}} f\left(t, u_{n}, u_{n}^{\prime \prime}\right) .
$$

We may assume that $\left\|u_{n}^{\prime \prime}\right\| \rightarrow \infty$ as $n \rightarrow \infty$. Let $v_{n}:=u_{n} /\left\|u_{n}^{\prime \prime}\right\|$. Then $v_{n}^{(r)}=u_{n}^{(r)} /\left\|u_{n}^{\prime \prime}\right\|$ for $r=0, \ldots, 4$. Thus, our sequence of equations has the form

$$
v_{n}^{(4)}-\left(\lambda+\varepsilon_{n}\right) v_{n}^{\prime \prime}-\left(\mu+\varepsilon_{n}\right) v_{n}=f_{n}(t),
$$

where $f_{n}:=\frac{1}{\left\|u_{n}^{\prime \prime}\right\|_{1}} f\left(\cdot, u_{n}, u_{n}^{\prime \prime}\right)$. It is obvious that

$$
f_{n} \rightrightarrows 0 \quad \text { as } n \rightarrow \infty \text {. }
$$

It is easily seen that $\left\|v_{n}^{\prime \prime}\right\|_{1}=1$, so $\left\{v_{n}^{\prime \prime}\right\}_{n \in \mathbb{N}}$ is bounded. The boundedness of $\left\{v_{n}^{(r)}\right\}_{n \in \mathbb{N}}$ for $r=0,1,3,4$ and the equicontinuity of $\left\{v_{n}^{(r)}\right\}_{n \in \mathbb{N}}$ for $r=$ $0,1,2,3$ follow by the same method as in the first step. Therefore, there exists a function $v$ such that $v_{n}^{(r)} \rightrightarrows v^{(r)}$ for $r=0,1,2,3$. We shall show that $v_{n}^{(4)} \rightrightarrows v^{(4)}$ as well. After passing to the limit in $v_{n}^{(4)}=\left(\lambda+\varepsilon_{n}\right) v_{n}^{\prime \prime}+$ $\left(\mu+\varepsilon_{n}\right) v_{n}+f_{n}(t)$, we have $\left.v_{n}^{(4)} \rightrightarrows \lambda v^{\prime \prime}+\mu v\left({ }^{1}\right)\right)$. Therefore, by applying the same arguments as above we finally have $v_{n}^{(r)} \rightrightarrows v^{(r)}$, where $r=1, \ldots, 4$ (considering a subsequence if necessary). Furthermore, the equality $\left\|v^{\prime \prime}\right\|_{1}$ $=1$ implies $\|v\|_{1} \neq 0$.

$\left({ }^{1}\right)$ Note that in the first step we have shown that $u_{n}^{(4)} \rightrightarrows u^{(4)}$ almost everywhere; now because of (16) we have $v_{n}^{(4)} \rightrightarrows v^{(4)}$ everywhere. 
Altogether, we can let $n \rightarrow \infty$ in (15) to obtain

$$
v^{(4)}-\lambda v^{\prime \prime}-\mu v=0 .
$$

This means that $v$ is the element of the eigenspace corresponding to $(\lambda, \mu)$ $\in \sigma_{1}^{2}$.

By multiplying (15) by $v$, we get

$$
v_{n}^{(4)} v-\left(\lambda+\varepsilon_{n}\right) v_{n}^{\prime \prime} v-\left(\mu+\varepsilon_{n}\right) v_{n} v=f_{n}(t) v .
$$

If we integrate this over $[0,1]$, we obtain

$$
\int_{0}^{1} v_{n}^{(4)} v d t-\left(\lambda+\varepsilon_{n}\right) \int_{0}^{1} v_{n}^{\prime \prime} v d t-\left(\mu+\varepsilon_{n}\right) \int_{0}^{1} v_{n} v d t=\int_{0}^{1} f_{n}(t) v d t .
$$

Integration by parts yields

$$
\int_{0}^{1} v_{n} v^{(4)} d t-\left(\lambda+\varepsilon_{n}\right) \int_{0}^{1} v_{n} v^{\prime \prime} d t-\left(\mu+\varepsilon_{n}\right) \int_{0}^{1} v_{n} v d t=\int_{0}^{1} f_{n}(t) v d t .
$$

Therefore

$$
\int_{0}^{1} v_{n}\left(v^{(4)}-\lambda v^{\prime \prime}-\mu v\right) d t-\varepsilon_{n} \int_{0}^{1} v_{n} v^{\prime \prime} d t-\varepsilon_{n} \int_{0}^{1} v_{n} v d t=\int_{0}^{1} f_{n}(t) v d t .
$$

By (17), the above equality takes the form

$$
-\varepsilon_{n} \int_{0}^{1} v_{n}\left(v^{\prime \prime}+v\right) \mathrm{dt}=\int_{0}^{1} f_{n}(t) v d t
$$

Analogously, multiplying (15) by $v^{\prime \prime}$ leads to

$$
-\varepsilon_{n} \int_{0}^{1} v_{n}^{\prime \prime}\left(v^{\prime \prime}+v\right) d t=\int_{0}^{1} f_{n}(t) v^{\prime \prime} d t
$$

Adding the above conditions, we obtain

$$
-\varepsilon_{n} \int_{0}^{1}\left(v_{n}+v_{n}^{\prime \prime}\right)\left(v+v^{\prime \prime}\right) d t=\int_{0}^{1} f_{n}(t)\left(v+v^{\prime \prime}\right) d t .
$$

Lebesgue's dominated convergence theorem implies that

$$
\lim _{n \rightarrow \infty} \int_{0}^{1}\left(v_{n}+v_{n}^{\prime \prime}\right)\left(v+v^{\prime \prime}\right) d t=\int_{0}^{1}\left(v+v^{\prime \prime}\right)^{2} d t .
$$

We have noticed that $v$ is not equal to 0 and satisfies (17). Therefore $v=$ $C \sin n_{0} \pi t$, where $C \neq 0$. Furthermore, $v^{\prime \prime}+v=\left(1-n_{0}^{2} \pi^{2}\right) C \sin n_{0} \pi t \neq 0$, so $v^{\prime \prime}+v \neq 0$. Hence the last equality gives

$$
\int_{0}^{1}\left(v+v^{\prime \prime}\right)^{2} d t>0 .
$$


This implies the existence of a natural number $N$ such that

$$
\int_{0}^{1}\left(v_{n}+v_{n}^{\prime \prime}\right)\left(v+v^{\prime \prime}\right) d t>0 \quad \text { for } n>N .
$$

On the one hand,

$$
v_{n}=\frac{u_{n}}{\left\|u_{n}^{\prime \prime}\right\|}, \quad v_{n}^{\prime \prime}=\frac{u_{n}^{\prime \prime}}{\left\|u_{n}^{\prime \prime}\right\|}
$$

and on the other

$$
\lim _{n \rightarrow \infty} v_{n}=C \sin n_{0} \pi t, \quad \lim _{n \rightarrow \infty} v_{n}^{\prime \prime}=-C n_{0}^{2} \pi^{2} \sin n_{0} \pi t .
$$

Consequently,

$$
\lim _{m \rightarrow \infty} \frac{u_{n}}{\left\|u_{n}^{\prime \prime}\right\|}=C \sin n_{0} \pi t, \quad \lim _{m \rightarrow \infty} \frac{u_{n}^{\prime \prime}}{\left\|u_{n}^{\prime \prime}\right\|}=-C n_{0}^{2} \pi^{2} \sin n_{0} \pi t .
$$

Let us consider two cases:

(a) If $t \in \Gamma^{+}$we conclude from (20) that

$$
\lim _{n \rightarrow \infty} u_{n}=\left\{\begin{array}{ll}
+\infty & \text { if } C>0, \\
-\infty & \text { if } C<0,
\end{array} \quad \lim _{n \rightarrow \infty} u_{n}^{\prime \prime}= \begin{cases}-\infty & \text { if } C>0, \\
+\infty & \text { if } C<0 .\end{cases}\right.
$$

Therefore

(21) $\quad \lim _{n \rightarrow \infty} f\left(t, u_{n}, u_{n}^{\prime \prime}\right)=\left\{\begin{array}{ll}f_{+}(t) & \text { if } C>0, \\ f_{-}(t) & \text { if } C<0,\end{array} \quad\right.$ for almost all $t \in \Gamma^{+}$.

(b) If $t \in \Gamma^{-}$we conclude from (20) that

$$
\lim _{n \rightarrow \infty} u_{n}=\left\{\begin{array}{ll}
-\infty & \text { if } C>0, \\
+\infty & \text { if } C<0,
\end{array} \quad \lim _{n \rightarrow \infty} u_{n}^{\prime \prime}= \begin{cases}+\infty & \text { if } C>0, \\
-\infty & \text { if } C<0 .\end{cases}\right.
$$

In this case

(22) $\lim _{n \rightarrow \infty} f\left(t, u_{n}, u_{n}^{\prime \prime}\right)=\left\{\begin{array}{ll}f_{-}(t) & \text { if } C>0, \\ f_{+}(t) & \text { if } C<0,\end{array} \quad\right.$ for almost all $t \in \Gamma^{-}$.

Consider the subsequences $\left\{\varepsilon_{n_{m}}\right\}_{m \in \mathbb{N}}$ and $\left\{\varepsilon_{n_{k}}\right\}$ of $\left\{\varepsilon_{n}\right\}_{n \in \mathbb{N}}$, where $\varepsilon_{n_{m}}=$ $1 /(2 m)$ and $\varepsilon_{n_{k}}=-1 /(2 k-1)$. Condition (19) implies that

$$
\begin{aligned}
-\varepsilon_{n_{m}} \int_{0}^{1}\left(v_{n_{m}}+v_{n_{m}}^{\prime \prime}\right)\left(v+v^{\prime \prime}\right) d t<0 & \text { for } m>N, \\
-\varepsilon_{n_{k}} \int_{0}^{1}\left(v_{n_{k}}+v_{n_{k}}^{\prime \prime}\right)\left(v+v^{\prime \prime}\right) d t>0 & \text { for } k>N .
\end{aligned}
$$


These inequalities together with (18) give

$$
\begin{array}{ll}
\int_{0}^{1} f\left(t, u_{n_{m}}, u_{n_{m}}^{\prime \prime}\right)\left(v+v^{\prime \prime}\right) d t<0 & \text { for } m>N, \\
\int_{0}^{1} f\left(t, u_{n_{k}}, u_{n_{k}}^{\prime \prime}\right)\left(v+v^{\prime \prime}\right) d t>0 & \text { for } k>N .
\end{array}
$$

Let $C>0$. Formula $(23)$ yields

$$
\begin{aligned}
0> & \int_{0}^{1} f\left(t, u_{n_{m}}, u_{n_{m}}^{\prime \prime}\right)\left(v^{\prime \prime}+v\right) d t \\
= & \int_{0}^{1} f\left(t, u_{n_{m}}, u_{n_{m}}^{\prime \prime}\right) C\left(1-n_{0}^{2} \pi^{2}\right) \sin n_{0} \pi t d t \\
= & C\left(\int_{\Gamma^{+}} f\left(t, u_{n_{m}}, u_{n_{m}}^{\prime \prime}\right)\left(1-n_{0}^{2} \pi^{2}\right) \sin n_{0} \pi t d t\right. \\
& \left.\quad+\int_{\Gamma^{-}} f\left(t, u_{n_{m}}, u_{n_{m}}^{\prime \prime}\right)\left(1-n_{0}^{2} \pi^{2}\right) \sin n_{0} \pi t d t\right) .
\end{aligned}
$$

It follows that

$$
\int_{\Gamma^{+}} f\left(t, u_{n_{m}}, u_{n_{m}}^{\prime \prime}\right) \sin n_{0} \pi t d t+\int_{\Gamma^{-}} f\left(t, u_{n_{m}}, u_{n_{m}}^{\prime \prime}\right) \sin n_{0} \pi t d t>0 .
$$

Letting $m \rightarrow \infty$ we get, by (21) and (22),

$$
\int_{\Gamma^{+}} f_{+}(t) \sin n_{0} \pi t d t+\int_{\Gamma^{-}} f_{-}(t) \sin n_{0} \pi t d t \geq 0 .
$$

If we proceed in the same way as above, but with respect to $\left\{\varepsilon_{n_{k}}\right\}$, we get from (24), (21) and (22)

$$
\int_{\Gamma^{+}} f_{+}(t) \sin n_{0} \pi t d t+\int_{\Gamma^{-}} f_{-}(t) \sin n_{0} \pi t d t \leq 0 .
$$

The inequalities (25) and (26) lead to

$$
\int_{\Gamma^{+}} f_{+}(t) \sin n_{0} \pi t d t+\int_{\Gamma^{-}} f_{-}(t) \sin n_{0} \pi t d t=0 .
$$

Let $C<0$. As in the previous case, we obtain

$$
\int_{\Gamma^{+}} f_{-}(t) \sin n_{0} \pi t d t+\int_{\Gamma^{-}} f_{+}(t) \sin n_{0} \pi t d t=0 .
$$

The equalities (27) and (28) contradict our assumptions. This proves (i).

If we substitute $f_{0}=f_{+}=f_{-}$in (27) and (28), we obtain

$$
\int_{\Gamma^{+} \cup \Gamma^{-}} f_{0}(t) \sin n_{0} \pi t d t=0
$$


regardless of the sign of $C$. This contradicts the assumption and proves (ii). This completes the proof.

Example 3.1. Let $\phi:[0,1] \rightarrow \mathbb{R}$ be continuous and positive. Consider the equation

$$
u^{(4)}+\frac{1}{\pi^{2}} u^{\prime \prime}+\left(1-\pi^{4}\right) u=\phi(t) \arctan \left(u^{2} u^{\prime \prime}-t\right)
$$

with the boundary conditions

$$
u(0)=u(1)=u^{\prime \prime}(0)=u^{\prime \prime}(1)=0 .
$$

This is a problem at resonance, with $n_{0}=1$. We have

$$
\begin{aligned}
& \lim _{\substack{x \rightarrow+\infty \\
y \rightarrow-\infty}} \phi(t) \arctan \left(x^{2} y-t\right)=-\frac{\pi}{2} \phi(t), \\
& \lim _{\substack{x \rightarrow-\infty \\
y \rightarrow+\infty}} \phi(t) \arctan \left(x^{2} y-t\right)=\frac{\pi}{2} \phi(t) .
\end{aligned}
$$

It is evident that in this case $\Gamma^{+}=[0,1]$ and $\Gamma^{-}=\emptyset$ and the LandesmanLazer conditions are satisfied. Theorem 3.1 implies that the above problem has a nontrivial solution.

Now, assume that $\lambda, \mu \in \mathbb{R}^{k}$ with $\left(\lambda^{j}, \mu^{j}\right) \in \sigma_{1}^{2}$, and there exists a positive integer $n_{0}$ such that $\mu^{j}=n_{0}^{2} \pi^{2} \lambda^{j}+n_{0}^{4} \pi^{4}$ for all $j=1, \ldots, k$. This means that the points $\left(\lambda^{j}, \mu^{j}\right)$ lie on the same line in the set $\sigma^{2}$.

Let

$$
\Gamma_{n_{0}}:=\left\{t \in[0,1] \mid \sin n_{0} \pi t \neq 0\right\} .
$$

We will write $\Gamma$ instead of $\Gamma_{n_{0}}$ (see Remark 3.1). It is apparent that the function $[0,1] \ni t \mapsto \sin n_{0} \pi t$ is the basis vector of the eigenspace corresponding to each $\left(\lambda^{j}, \mu^{j}\right) \in \sigma_{1}^{2}$. This space will be denoted by $\mathcal{V}_{0}$.

Theorem 3.2. Let $f:[0,1] \times \mathbb{R}^{k} \times \mathbb{R}^{k} \rightarrow \mathbb{R}^{k}$ be a bounded Carathéodory map and suppose that

$$
\lim _{\langle x, y\rangle \rightarrow-\infty} f^{j}(t, x, y)=f_{0}^{j}(t), \quad j=1, \ldots, k,
$$

for almost all $t \in[0,1]$. If for each $j=1, \ldots, k$,

$$
\int_{0}^{1} f_{0}^{j}(t) \sin n_{0} \pi t d t \neq 0,
$$

then the problem (1) has a solution.

Proof. Set

$$
\varepsilon_{n}^{j}:= \begin{cases}1 / n & \text { if } n \text { is even, } \\ -1 / n & \text { if } n \text { is odd }\end{cases}
$$


for $j=1, \ldots, k$. Put $\varepsilon_{n}:=\left[\varepsilon_{n}^{1}, \ldots, \varepsilon_{n}^{k}\right]$ and consider the sequence of boundary value problems

$$
\begin{aligned}
& x^{(4)}-\left(\lambda+\varepsilon_{n}\right) \bullet x^{\prime \prime}-\left(\mu+\varepsilon_{n}\right) \bullet x=f\left(t, x(t), x^{\prime \prime}(t)\right), \\
& x(0)=x(1)=x^{\prime \prime}(0)=x^{\prime \prime}(1)=0 .
\end{aligned}
$$

Theorem 2.1 implies that for every $n \in \mathbb{N}$ there exists a solution $u_{n}$ to the above problem. In the same way as above we show that if the sequence $\left\{u_{n}\right\}_{n \in \mathbb{N}}$ is bounded then it has a subsequence convergent to a solution of (1).

Now, suppose that $\left\{u_{n}^{\prime \prime}\right\}_{n \in \mathbb{N}}$ is not bounded. By using the same method as in Theorem 2.1 we obtain a sequence $\left\{v_{n}\right\}_{n \in \mathbb{N}}$ such that $v_{n}^{j}=u_{n}^{j} /\left\|u_{n}^{\prime \prime}\right\|_{k}$, $\left\|v_{n}^{\prime \prime}\right\|_{k}=1$ and

$$
v_{n}^{(4)}-\left(\lambda+\varepsilon_{n}\right) \bullet v_{n}^{\prime \prime}-\left(\mu+\varepsilon_{n}\right) \bullet v_{n}=f_{n}(t),
$$

where $f_{n}:=\frac{1}{\left\|u_{n}^{\prime \prime}\right\|_{k}} f\left(\cdot, u_{n}, u_{n}^{\prime \prime}\right)$. Furthermore, $v_{n}^{(s)} \rightrightarrows v^{(s)}$, where $s=0, \ldots, 4$, $v \neq 0$ and

$$
v^{(4)}-\lambda \bullet v^{\prime \prime}-\mu \bullet v=0 .
$$

This means that $v^{j}, j=1, \ldots, k$, is an element of the space $\mathcal{V}_{0}$. It is obvious that $v=\left[v_{1}, \ldots, v_{k}\right]$ is not equal to 0 and satisfies (30). Therefore, there exists at least one $j$ such that $v^{j}=C^{j} \sin n_{0} \pi t$, where $C^{j} \neq 0$. Define $V:=\left\{j \in\{1, \ldots, k\} \mid C^{j} \neq 0\right\}$. Then for $j \in V$ we have $\left(v^{j}\right)^{\prime \prime}+v^{j}=$ $\left(1-n_{0}^{2} \pi^{2}\right) C^{j} \sin n_{0} \pi t \neq 0$, thus $v^{\prime \prime}+v \neq 0$.

On the one hand, we have

$$
\left\langle v_{n}, v_{n}^{\prime \prime}\right\rangle=\frac{1}{\left\|u_{n}^{\prime \prime}\right\|_{k}^{2}}\left\langle u_{n}, u_{n}^{\prime \prime}\right\rangle,
$$

and on the other,

$$
\lim _{n \rightarrow \infty} v_{n}=\sin n_{0} \pi t \cdot C, \quad \lim _{n \rightarrow \infty} v_{n}^{\prime \prime}=-n_{0}^{2} \pi^{2} \sin n_{0} \pi t \cdot C,
$$

where $C=\left[C^{1}, \ldots, C^{k}\right]$. Therefore

$$
\lim _{n \rightarrow \infty}\left\langle v_{n}, v_{n}^{\prime \prime}\right\rangle=-n_{0}^{2} \pi^{2} \sin ^{2} n_{0} \pi t\|C\|^{2} .
$$

We have $\|C\| \neq 0$, so for $t \in \Gamma$,

$$
\lim _{n \rightarrow \infty}\left\langle u_{n}, u_{n}^{\prime \prime}\right\rangle=-\infty
$$

This implies that for $t \in \Gamma$,

$$
\lim _{n \rightarrow \infty} f^{j}\left(t, u_{n}, u_{n}^{\prime \prime}\right)=f_{0}^{j}(t) .
$$

Let $j \in V$. Consider the subsequences $\left\{\varepsilon_{n_{m}}^{j}\right\}_{m \in \mathbb{N}}$ and $\left\{\varepsilon_{n_{k}}^{j}\right\}_{k \in \mathbb{N}}$ of $\left\{\varepsilon_{n}^{j}\right\}_{n \in \mathbb{N}}$, where $\varepsilon_{n_{m}}^{j}=1 /(2 m)$ and $\varepsilon_{n_{k}}^{j}=-1 /(2 k-1)$. As in the proof of the previous 
theorem, we can show that there exists $N$ such that

$$
\begin{array}{cc}
C^{j} \int_{\Gamma} f^{j}\left(t, u_{n_{m}}, u_{n_{m}}^{\prime \prime}\right) \sin n_{0} \pi t d t>0 & \text { for } m>N, \\
C^{j} \int_{\Gamma} f^{j}\left(t, u_{n_{k}}, u_{n_{k}}^{\prime \prime}\right) \sin n_{0} \pi t d t<0 & \text { for } k>N .
\end{array}
$$

If we reason as in the proof of Theorem 3.1, we obtain

$$
\int_{\Gamma} f_{0}^{j}(t) \sin n_{0} \pi t d t=0,
$$

regardless of the sign of $C^{j}, j \in V$. This contradiction implies that there exists a solution to problem (1). This completes the proof.

REMARK 3.2. It has to be emphasized that, in particular for $k=1$, the limit condition in the last theorem has the form

$$
\lim _{x \cdot y \rightarrow-\infty} f(t, x, y)=f_{0}(t) \quad \text { for almost all } t \in[0,1] .
$$

It is easily seen that this condition is stronger than the corresponding one from Theorem 3.1(b).

EXAMPLE 3.2. Let $\psi: \mathbb{R}^{4} \rightarrow \mathbb{R}$ be a continuous function satisfying the condition $\psi(x, y) \leq\langle x, y\rangle\left(x=\left[x_{1}, x_{2}\right], y=\left[y_{1}, y_{2}\right]\right)$ and let $\varphi: \mathbb{R} \rightarrow \mathbb{R}$ be given by the formula

$$
\varphi(t):= \begin{cases}\varphi_{1}(t) & \text { for } t \in[0,1 / 3], \\ -\varphi_{2}(t) & \text { for } t \in(1 / 3,2 / 3), \\ \varphi_{3}(t) & \text { for } t \in[2 / 3,1],\end{cases}
$$

where $\varphi_{1}, \varphi_{2}, \varphi_{3}$ are continuous and positive. Consider the system of BVP

$$
\begin{aligned}
x_{1}^{(4)}(t)-\frac{1}{9 \pi^{2}} x_{1}^{\prime \prime}(t)- & x_{1}(t)-81 \pi^{4} x_{1}(t) \\
& =\varphi(t)\left(\frac{\psi\left(x_{1}(t), x_{2}(t), x_{1}^{\prime \prime}(t), x_{2}^{\prime \prime}(t)\right)}{1+\left[\psi\left(x_{1}(t), x_{2}(t), x_{1}^{\prime \prime}(t), x_{2}^{\prime \prime}(t)\right)\right]^{2}}+1\right), \\
x_{1}^{(4)}(t)+\pi^{2} x_{1}^{\prime \prime}(t)- & 72 \pi^{4} x_{1}(t)=t \arctan \left(x_{1}(t) x_{1}^{\prime \prime}(t)+x_{2}(t) x_{2}^{\prime \prime}(t)\right),
\end{aligned}
$$

with the boundary conditions

$$
\begin{aligned}
& x_{1}(0)=x_{1}(1)=x_{1}^{\prime \prime}(0)=x_{1}^{\prime \prime}(1)=0, \\
& x_{2}(0)=x_{2}(1)=x_{2}^{\prime \prime}(0)=x_{2}^{\prime \prime}(1)=0 .
\end{aligned}
$$

This is a resonance problem because $\left(1 /\left(9 \pi^{2}\right), 1+81 \pi^{4}\right),\left(-\pi^{2}, 72 \pi^{4}\right) \in \sigma_{1}^{2}$. Furthermore, it is apparent that these points lie on the line $\mu=9 \pi^{2} \lambda+81 \pi^{4}$. We have

$$
\lim _{\langle x, y\rangle \rightarrow-\infty} t \arctan (\langle x, y\rangle)=-\frac{\pi}{2} t .
$$


The inequality $\psi(x, y) \leq\langle x, y\rangle$ implies that

$$
\lim _{\langle x, y\rangle \rightarrow-\infty} \varphi(t)\left(\frac{\psi\left(x_{1}, x_{2}, y_{1}, y_{2}\right)}{1+\left[\psi\left(x_{1}, x_{2}, y_{1}, y_{2}\right)\right]^{2}}+1\right)=\varphi(t) .
$$

Therefore

$$
-\int_{0}^{1} \frac{\pi}{2} t \sin 3 \pi t d t=-\frac{1}{6} \neq 0
$$

and

$$
\int_{0}^{1} \varphi(t) \sin 3 \pi t d t \neq 0,
$$

because of (33). The above conditions and Theorem 3.2 lead to the conclusion that the above problem has a solution.

Acknowledgements. The author would like to thank the anonymous referee for helpful remarks that made it possible to eliminate an unclear and useless theorem.

\section{References}

[1] J. Dugundji and A. Granas, Fixed Point Theory, PWN, Warszawa, 1981.

[2] G. Han and Z. Xu, Multiple solutions of some nonlinear fourth-order beam equations, Nonlinear Anal. 68 (2008), 3646-3656.

[3] F. Li, Q. Zhang and Z. Liang, Existence and multiplicity of solutions of a kind of fourth-order boundary value problem, ibid. 62 (2005), 803-816.

[4] Y. Li, Positive solutions of fourth-order boundary value problems with two parameters, J. Math. Anal. Appl. 281 (2003), 477-484.

[5] —, Positive solutions of fourth-order periodic boundary value problems, Nonlinear Anal. 54 (2003), 1069-1078.

[6] X.-L. Liu and W.-T. Li, Positive solutions of the nonlinear fourth-order beam equation with three parameters, J. Math. Anal. Appl. 303 (2005), 150-163.

[7] E. M. Landesman and A. C. Lazer, Nonlinear perturbations of linear elliptic boundary value problems at resonance, J. Math. Mech. 19 (1970), 609-623.

[8] B. Przeradzki, A new continuation method for the study of nonlinear equations at resonance, J. Math. Anal. Appl. 180 (1993), 553-565.

[9] J. Przybycin, Existence and uniqueness theorem for fourth-order boundary value problems, Ann. Polon. Math. 67 (1997), 59-64.

Faculty of Mathematics and Computer Science

University of Łódź

Banacha 22

90-238 Łódź, Poland

E-mail: mjurkiewicz@math.uni.lodz.pl 